\title{
The FAST telescope and its possible contribution to high precision astrometry
}

\author{
C. J. Jin, R. D. Nan and H. Q. Gan \\ National Astronomical Observatories, Chinese Academy of Sciences \\ Chaoyang District, Datun Road, A.20, Beijing 100012, China \\ email: cjjin@bao.ac.cn
}

\begin{abstract}
In this report we give a brief introduction to the Five hundred meter Apeture Spherical Telescope (FAST). Some possible contributions of FAST to high precision astrometry are discussed. The illuminated aperture of FAST in normal operation mode is $300 \mathrm{~m}$ in diameter. With special feeding mechanism, the whole $500 \mathrm{~m}$ aperture could be used. FAST will cover frequencies from $70 \mathrm{MHz}$ to $3 \mathrm{GHz}$, and observe at zenith angle of up to 40 degrees without a notable gain loss. As the most sensitive single dish radio telescope, FAST would be able to discover more mega-masers and measure the radial velocities of masers with higher precision. This may yield more delicate dynamics of their maser spots. FAST will increase the precision of time of arrival (ToA) measurements for pulsars. This will help in detecting the stochastic gravitational wave background and in establishing an independent timing standard based on the long-term stability of the rotations of a group of millisecond pulsars. FAST might also work as a very powerful ground station for the future space missions. In a three-way communication mode, FAST should be able to provide precise ranging and Doppler measurements. Moreover, by joining the international VLBI network, FAST would help to improve the precision of the VLBI astrometry measurements.
\end{abstract}

Keywords. telescopes, masers, pulsars: general, gravitational waves, reference systems

\section{Introduction}

FAST, the Five hundred meter Aperture Spherical Telescope, is one of the mega science facilities for fundamental research in China (Nan 2006). After more than 13 years of studies of its science case and key technologies, funding for the FAST project was approved by the National Development and Reform Commission (NDRC) in July, 2007. The construction of FAST is expected to be completed around 2014. FAST will be about 10 times more sensitive than the fully steerable $100 \mathrm{~m}$ telescope Effelsberg and GBT. Compared with the $305 \mathrm{~m}$ Arecibo telescope, FAST will be about 3 times more sensitive, and has a much wider sky coverage. As the largest single dish radio telescope ever built, FAST would help to carry out astrometric observations with unprecedented sensitivity.

In the following section we describe the general specifications and key technologies of FAST. Some possible contributions of FAST to high precision astrometry are discussed in the third section. A concluding remark is drawn in the last section.

\section{The general specifications and key technologies}

Figure. 1 shows a 3-D image of the FAST telescope and its optics. FAST will be built in a Karst depression. The terrain shown in Fig. 1 is taken from the real depression where FAST will be built. The shape of the main reflector is spherical with a radius of $300 \mathrm{~m}$. The overall diameter is $500 \mathrm{~m}$. The focus cabin is suspended about $140 \mathrm{~m}$ above the reflector. During observations, part of the main reflector will be deformed instantaneously into a 


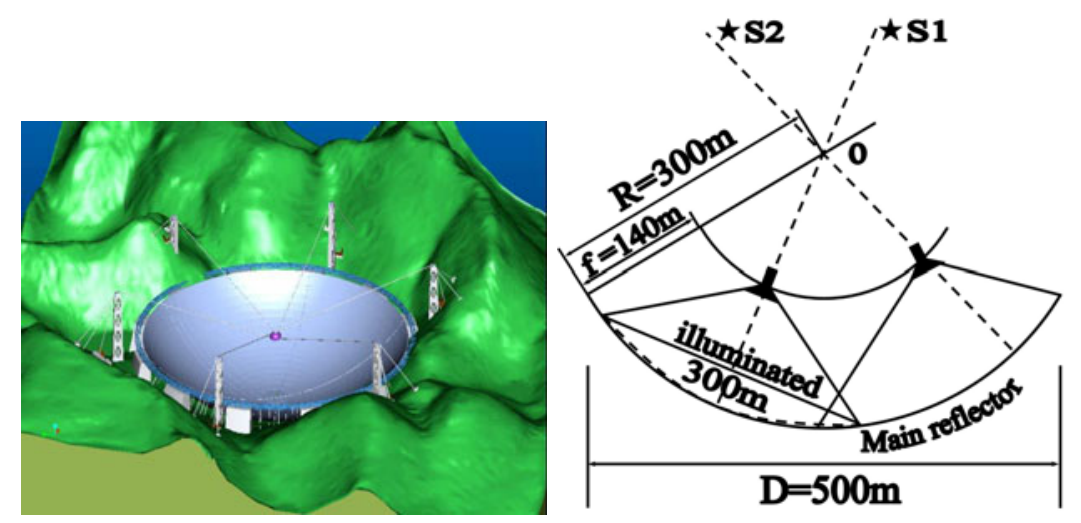

Figure 1. A 3-D image of the FAST telescope (left) and its optics (right). Note that the terrain in the left image is taken from the real depression at Dawodang.

Table 1. The main specifications of FAST telescope.

\begin{tabular}{|c|c|}
\hline Item & Specification \\
\hline Spherical reflector & | Radius $\mathbf{3 0 0 m}$, Aperture $\mathbf{5 0 0 m}$, Opening angle $110 \sim 120^{\circ}$ \\
\hline Illuminated aperture & $D_{i l l u}=300 \mathrm{~m}$ \\
\hline Focal ratio & $\mathrm{f} / \mathrm{D}=0.4665$ \\
\hline Sky coverage & zenith angle $40^{\circ}$ \\
\hline Frequency & $70 \sim 3000 \mathrm{M} \mathrm{Hz}$ \\
\hline Multi-beam(L-band) & 19 , beam number of future FPA $>100$ \\
\hline Slewing & $<10 \mathrm{~min}$ \\
\hline Pointing accuracy & $8^{\prime \prime}$ \\
\hline
\end{tabular}

paraboloid through active control. This illuminated part has a diameter of $300 \mathrm{~m}$. The optics of FAST allows the use of standard feed design. With special feeding mechanism, the whole $500 \mathrm{~m}$ aperture can be illuminated. FAST will cover frequencies from $70 \mathrm{MHz}$ to $3 \mathrm{GHz}$, and it will observe at a zenith angle up to $40 \mathrm{deg}$ without a notable gain loss. The main specifications of FAST is listed in Table 1

The technical studies of FAST have been focused on 5 key technologies: the site, active main reflector, focus cabin suspension, measurement \& control, and receivers.

$\underline{\text { Site. }}$. An extensive site survey was carried out in the Karst region in Guizhou province (Nan et al. 2002). After evaluation on the various aspects of depressions, including the shape, distribution of surrounding peaks, natural sink holes for drainage and RFI situations, Dawodang depression $\left(N 25.647222^{\circ}, E 106.85583^{\circ}\right)$ is finally selected as the site for FAST.

Active main reflector. The central part of sphere is close to paraboloid as the focal length is properly chosen. In the case of FAST, the radial deviation between the sphere and the paraboloid is less than $0.67 \mathrm{~m}$ across the illuminated area (Qiu 1998). The back structure of the main reflector is made from steel cables. The elasticity of the cable is used to implement the required deformations (Nan et al. 2003).

Focus cabin suspension. During observations, the feed needs to be located at the focus of the instantaneous paraboloid with high accuracy. A coarse drive by cables and a secondary fine adjusting stewart platform are utilized to achieve the desired positional 
accuracy. Extensive theoretical study and model experiments were carried out on the focus cabin suspension system (Zhu et al. 2004).

Measurement $\&$ control. The shape of the main reflector and the position of the feed need to be monitored and controlled in real time. This involves monitoring the position of the feed at long distance with high sampling rate and surveying large number of nodes on the main reflector with relatively low sampling rate. Fusion of various measuring techniques is adopted for FAST (Zhu et al. 2004).

Receiver. The optics of FAST allows the use of standard feed design. In 2000, a layout design of receivers for FAST was made jointly by the National Astronomical Observatories, Chinese Academy of Sciences (NAOC) and Jodrell Bank Observatory (JBO). The layout was based on the existing proven design at the various frequency bands. In 2006, this layout was upgraded. An optimization of the band division and receiver design is still ongoing, to make the best match to the science drivers.

\section{The possible contributions to high precision astrometry}

As the largest single dish radio telescope ever built, FAST would observe the radio sky with unprecedented sensitivity.

Masers. Astronomical masers associated with stars and active galactic nuclei provide useful probes to study the dynamics of them (e.g., Reid \& Moran 1981; Lo 2005). In some cases, maser observations could yield distance measurement by trigonometric technique. The shells of $\mathrm{OH} 1612 \mathrm{MHz}$ emission surrounding OH-IR sources offer such a possibility. The light-travel diameter of the shell could be inferred by measuring phase-lags in the $\mathrm{OH}$ spectrum, but the angular diameter is measured by radio interferometry. The distance is then obtained geometrically (Shepherd et al. 1992). Such IR-OH sources would then provide an independent calibration to the period-luminosity of the RR Lyrae that belongs to the same system.

Pulsars. Pulsars are cosmic clocks that provide ultra stable periodic pulses. The stability of some of the millisecond pulsars are competitive to or even better than the most stable atomic clocks. A group of millisecond pulsars well-distributed on the sky may provide an independent timing standard that could complement the atomic standard. Long-term monitoring of the ToA of a group of millisecond pulsars would also help in detecting the stochastic gravitational wave background. The high sensitivity and large sky coverage make FAST a powerful tool for discovering more pulsars and measuring the ToA of pulsars with much better precision. A more detailed discussion on observing pulsars using FAST could be found in Nan et al. (2006).

Ground station for space mission. FAST might also work as a very powerful ground station for the future space missions. The large collecting area will enable the downlink data rate increase by orders of magnitude. In three-way communication mode, FAST would be able to provide more precise ranging and Doppler measurements. In the case of a flyby event, FAST would then allow a better mass determination of the flyby object.

VLBI element. Due to its large collecting area and geographical location, FAST could greatly improve the capability of the current international VLBI network. FAST lies at the edge of most of the VLBI networks. Figure 2 shows the simulated uv-coverage of some of EVN antennas plus FAST. It is clearly seen that FAST contributes mostly to long baselines. With an aperture of $300 \mathrm{~m}$, FAST would increase the baseline detection sensitivity by an order of magnitude. This may yield more precise position measurement and weaker sources to be detected. Although in current design the highest frequency of FAST is $3 \mathrm{GHz}$, it is feasible to extend it to $\mathrm{X}$ band when more delicate measurement and 

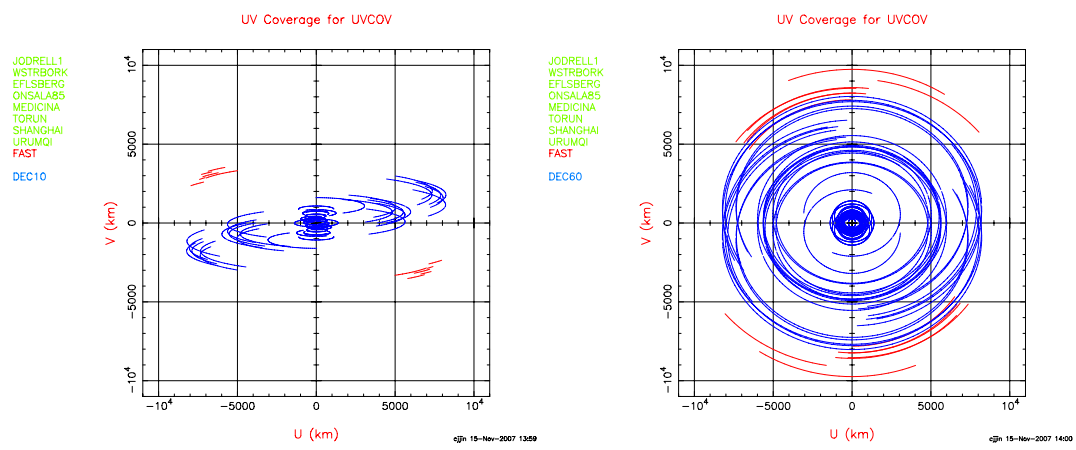

Figure 2. The simulated uv-coverage of EVN+FAST for sources of Declination $10^{\circ}$ and $60^{\circ}$

control mechanisms are employed in the future. This will enable FAST to participate in the $\mathrm{S} / \mathrm{X}$ astrometric and geodetic VLBI measurements.

\section{Concluding remarks}

FAST is going to be the largest and the most sensitive single dish radio telescope in the world. Because of its large collecting area, FAST will be able to carry out astronomical observations with unprecedented sensitivity. Although FAST is not a dedicated astrometric telescope, it will be able to contribute to high precision astrometry through single dish observations of masers and pulsars and by joining the S/X astrometric and geodetic VLBI network.

\section{Acknowledgements}

The authors thank the SOC of IAUS248 for the acceptance of this presentation. We also thank Mark Reid, Chopo Ma and Xingwu Zheng for the valuable discussions on the FAST-related topics during the symposium. This work is supported by the Chinese Academy of Sciences and a key project grant 10433020 from the National Natural Science Foundation in China.

\section{References}

Lo, K. Y. 2005, ARAA, 43, 625

Nan, R. D., Peng, B., Su, Y., Zhu, W. B., Zhu, L. C., Qiu, Y. H., Zhu, B. Q., \& Nie, Y. P. 2002, in: J. Vernin, Z. Benkhaldoun \& C. Muñoz-Tuñón(eds.), Astronomical Site Evaluation in the Visible and Radio Range, Proc. ASP, Vol. 266, p. 408

Nan, R. D., Ren G. X., Zhu, W. B., \& Lu, Y. J. 2003, Radio Studies of Galactic Objects, Galaxies and AGNs Proc. Sino-German Radio Astronomy Conference Radio Studies of Galactic Objects, Acta Astronomica Sinica, Supplement Issue, Vol. 44, p. 13

Nan, R. D. 2006, Science in China Series G Physics, Mechanics \& Astronomy, 49(2), 129

Nan, R. D., Wang, Q. M., Zhu, L. C., Zhu, W. B., Jin, C. J., \& Gan, H. Q. 2006, in: N. Wang, R. N. Manchester, B. J. Richett \& A. Esamdin (eds.), ChJAAS Vol. 6, Issue S2, Proc. 2005 Lake Hanas International Pulsar Symposium, p. 304

Qiu, Y. H. 1998, MNRAS, 301, 827

Reid, M. J. \& Moran, J. M. 1981, ARAA, 19, 231

Shepherd, M. C., Cohen, R. J., Gaylard, M. J., \& West, M. E. 1992, in: C. de Jager, \& H. Nieuwenhuijzen (eds.), Proc. International Colloquium, Amsterdam, p. 78

Zhu, W. B., Nan, R. D., \& Ren, G. X. 2004, Experimental Astronomy, Vol. 17, Issue 1-3, p. 177

Zhu, L. C., Nan, R. D., Wu, S. B., Zhu, W. B., \& Wang, Q. M. 2004, in: H. Lewis \& G. Raffi (eds.), Advanced Software, Control, and Communication System for Astronomy, Proc. SPIE, Vol. 5496 , p. 335 